\section{Aktuelle und künftige Entwicklung des Frühfördersystems in Schleswig-Holstein}

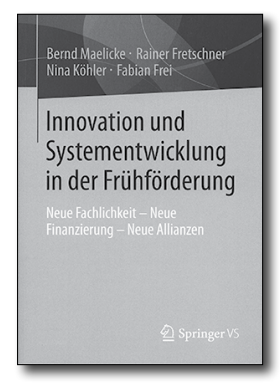

Bernd Maelicke, Rainer

Fretschner, Nina Köhler,

Fabian Frei

Innovation und

Systementwicklung

in der Frühförderung

Neue Fachlichkeit -

Neue Finanzierung -

Neue Allianzen

Wiesbaden: Springer, 2013, 209 S., $€ 34,99$,

ISBN 978-3658029302

Die Systementwicklung und Innovation in der Frühförderung lässt sich anhand des dargestellten Gutachtens eines umfassenden Projektes über die Frühförderung in Schleswig-Holstein von 2010-2012, ausgeschrieben vom dortigen Ministerium für Soziales, Gesundheit, Familie und Gleichstellung, beispielhaft für das Land Schleswig-Holstein erfahren. Da es sich u.a. um Vollerhebungen bei Experten sowie Fokusgruppen handelt, die Vielfalt der Akteure der Frühförderung berücksichtigt wurde und in den Beirat des Gutachtens alle für die Frühförderung in Schleswig-Holstein relevanten Organisationen und Institutionen einbezogen worden sind, ergibt diese breite Aufstellung einen interessanten Ansatz. Das sich in den regionalen Spezifika sehr differenziert zeigende Frühfördersystem soll in seinem Wandel gekennzeichnet und auch an der schrittweisen Verbesserung der Rahmenbedingungen für das Frühfördersystem gearbeitet werden. Das Gutachten empfiehlt eine landesweite Steuerung/Strukturierung (durch eine neue Landesrahmenvereinbarung für das Gesamtsystem und die Teilsysteme der Frühförderung im Hinblick auf die verbindliche bessere Erfassung von Daten als Basis für Veränderungen der rechtlichen und finanziellen Rahmenbedingungen). Dargestellt werden von der Autorengruppe zunächst der auch für andere Bundesländer wahrnehmbare
Strukturwandel in der Gruppe der Förderkinder und die Bedeutung psychosozialer Risiken sowie multiaxialer Belastungsfaktoren vor dem Hintergrund des Wandels der traditionell-bürgerlichen zur Risikogesellschaft. Außerdem auch andernorts relevant ist die statistisch hinterlegte zu späte oder mangelnde Vorstellung von Kindern mit Bedarf zur Frühförderung. Da die (kommunalen) Leistungsträger der Finanzierung von Frühförderung einen administrativen Anhaltspunkt zur Einschätzung einer "drohenden Behinderung" brauchen, liegt in der alltäglichen Praxis immer noch der Fokus auf der "Störung" des Kindes entgegen theoretischen wissenschaftlichen Perspektiven. Auch für die Eltern stehen noch die Störung des Kindes und seine heilpädagogische "Übungsbehandlung" im Vordergrund, statt die Stärkung der Gesamtressourcen der risikobelasteten Familie. Der Anspruch der Komplexleistung des Rehagesetzes steht der Veränderungsresistenz in der Verwaltung entgegen. Es werden Konkurrenzsituationen zwischen verschiedenen Anbietern auf einem wirtschaftlich orientierten Sozialmarkt gefördert, was fachlichen Verbundsystemen und systematischer Kooperation entgegenwirkt. Diese Spaltung hat mit den Zielen der Ressourcenorientierung und Interdisziplinarität wenig zu tun, wird vermerkt. Festgestellt wird, dass für die Abstimmung zwischen den Fachpersonen der verschiedenen Hilfe-Systeme (Jugendhilfe, Behindertenhilfe, Therapeuten) keine Finanzierungsstrukturen geschaffen worden sind (z. B. zur Kooperation mit einem externen Therapeuten durch die Frühförderstellen). Schließlich wird darauf hingewiesen, dass Effektivitätsstudien bereits weltweit belegen, dass der Erfolg der Einzelförderung des Kindes in der Frühförderung gering ist, wenn sie nicht in ein familienorientiertes Gesamtkonzept eingebettet wird. Nach dieser Bestandsaufnahme erfolgt ein Überblick über die Systementwicklung von den 70er-Jahren bis heute, über die gängigen Arbeitsprinzipien, Ziele und Organisationsformen der Frühförderung. Der Unterschied zwischen Frühförderung 
und Frühen Hilfen wird anhand einzelner Leistungsformen erörtert. Interessant liest sich, wie in Schleswig-Holstein die spezifische Frühförderung und die Fachleistungsstunde im Einzelnen organisiert sind. Gegen Schluss ist leider der Punkt der Wirtschaftlichkeit der Frühförderstellen zu kurz präsentiert worden. In einem letzten Kapitel wird abrundend auf die Systeme der
Frühförderung anderer Bundesländer eingegangen. Es folgen schließlich als Konsequenzen des Gutachtens Vorschläge und Empfehlungen zur Weiterentwicklung des Frühfördersystems in Schleswig-Holstein.

Dr. Doreen Kolaschinsky, Potsdam DOI 10.2378/fi2017.art15d 